\title{
La gestion technique centralisée des installations locales et distantes par moyens informatiques
}

\author{
J.P. Hennegrave, M.C. Huau
}

SAUR Challenger, 1, avenue Eugène-Freyssinet, 78064 St-Quentin-en-Yvelines

\section{Introduction}

La complexité de plus en plus accrue de la gestion d'exploitation des installations de traitement d'eau incite à développer des outils puissants et fiables, capables d'assurer un contrôle plus rigoureux de toutes les étapes du traitement, de permettre un pilotage complet adapté aux conditions changeantes environnantes. Aujourd'hui, si les automates locaux sont répandus sur les installations, l'exploitation, la veille et l'identification des anomalies reposent encore en grande partie sur les opérateurs. L'informatique industrielle est un outil moderne de gestion qui répond à ces préoccupations en effectuant les fonctions de surveillance et de télégestion. Cet outil a été mis en oeuvre dans l'usine de production d'eau potable de BasseGoulaine du Syndicat mixte Sud-Est de la LoireAtlantique lors de vastes travaux de renouvellement. Après une présentation rapide de l'usine, l'architecture du système informatique sera développée et les différentes étapes d'informatisation avec les fonctionnalités associées seront décrites.

\section{L'usine de production d'eau potable de Basse- Goulaine}

Cette installation a connu depuis sa première mise en service en 1952 une évolution importante de sa capacité. Gérée par le Syndicat mixte du Sud-Est de la Loire Atlantique, l'usine a eu deux étapes successives de renforcement de sa capacité passant de 3000 à $30000 \mathrm{~m}^{3} / \mathrm{j}$ en 1971 et la construction d'une deuxième tranche de $30000 \mathrm{~m}^{3} / \mathrm{j}$ en 1978. La capacité actuelle est donc de $60000 \mathrm{~m}^{3} / \mathrm{j}$ pour une desserte de 350000 habitants dans la périphérie de Nantes et de la région du Nord - Vendéen.

Le traitement s'opère en deux filières parallèles de 30000 $\mathrm{m}^{3} / \mathrm{j}$ chacune. Le traitement est classique et présenté dans la figure ci-dessous. Il permet l'élimination du fer, du manganèse et de l'ammoniac présents dans l'eau brute avec des teneurs respectives moyennes de $5 \mathrm{mg} / \mathrm{l}, 1 \mathrm{mg} / \mathrm{l}$ et 1 $\mathrm{mg} / \mathrm{l}$. L'eau brute pompée par forage sous la nappe alluviale de la Loire est traitée puis stockée dans cinq bâches au sol d'une capacité totale de $7200 \mathrm{~m}^{3}$ et refoulée par pompes dans cinq directions.

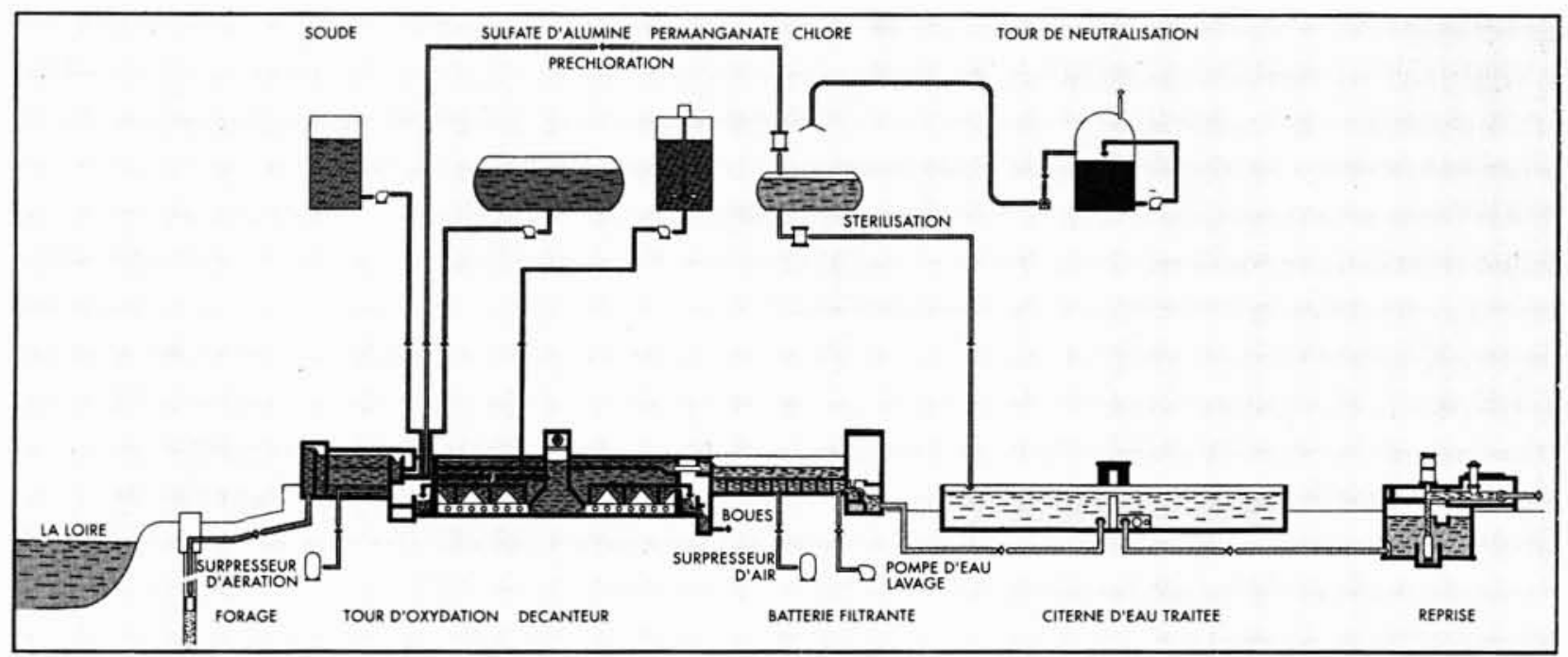




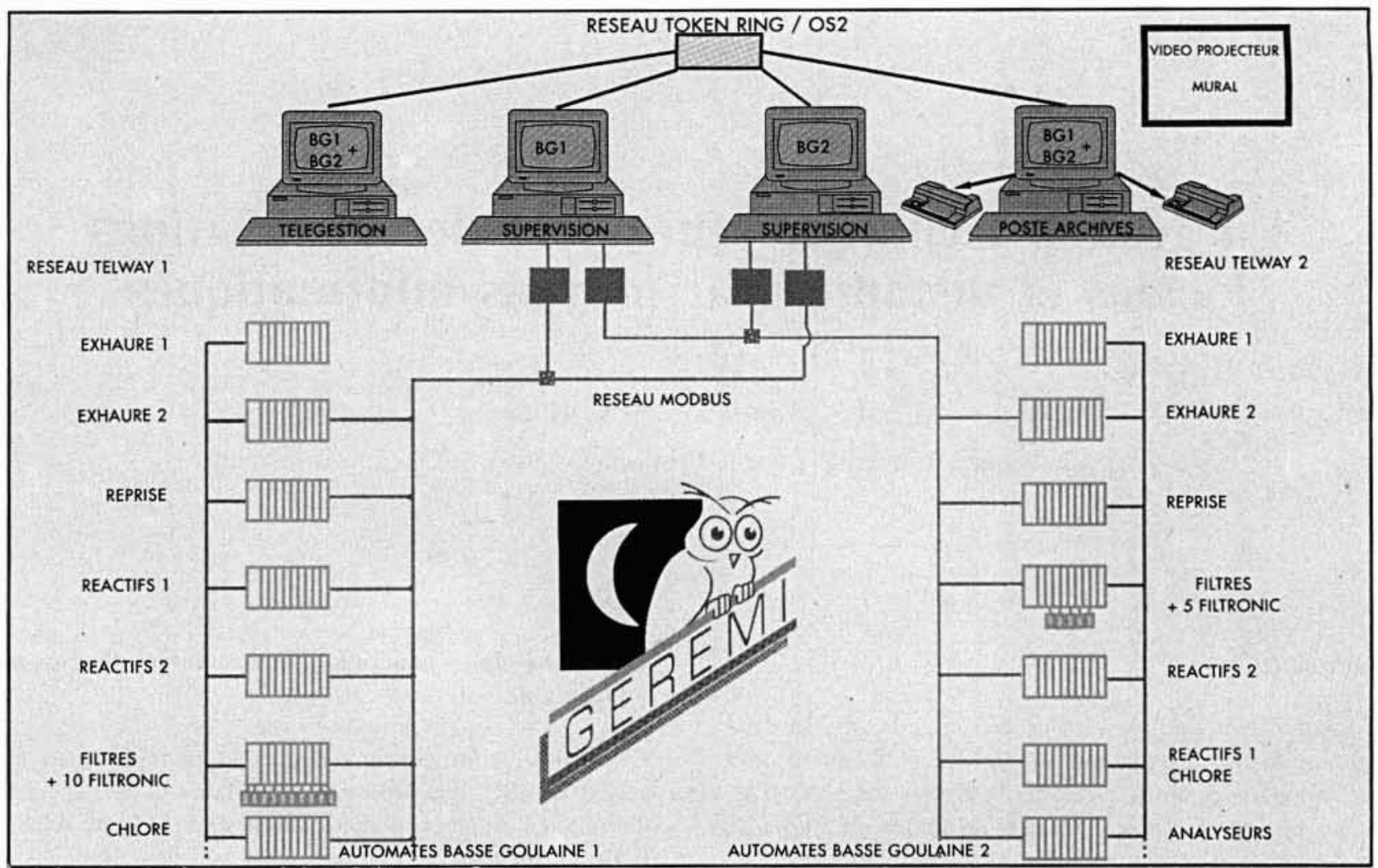

\section{L'architecture du système informatique}

Sans modifier la filière de traitement initiale, l'objet de la modernisation à l'aide de l'informatique industrielle était d'avoir un outil de surveillance, de supervision et de télégestion de l'installation pour aider l'exploitant dans la conduite et la gestion globale de son usine.

Afin d'assurer une cohérence de l'ensemble, un schéma directeur informatique complet a été élaboré. Il intègre ainsi successivement cinq étapes complémentaires d'informatisation de l'installation :

- l'automatisation locale de toutes les étapes de production ;

- la supervision globale de l'usine;

— la mise en place d'équipements satellites périphériques ;

- la télégestion des satellites ;

- le poste central d'archivage et d'aide à la gestion d'exploitation.

Ainsi, l'architecture de l'ensemble du système d'information s'est modifiée. Elle est à présent éclatée suivant le fil de l'eau tout en maintenant un contrôle local centralisé.

La communication des informations acquises dans ce système est de type " multiprotocole » et " multisupport ». Trois réseaux de communication ont été développés pour faire fonctionner le système d'information :

- un réseau primaire Token ring du constructeur IBM, qui est un réseau à grande vitesse dont la fonction est le transfert de fichiers entre les postes de supervision, télégestion et central d'archives;
- deux réseaux secondaires Modbus (protocole Gould Modicon), réseaux locaux industriels standards dont la fonction est de rapatrier l'information acquise entre les automates et les superviseurs ;

- deux réseaux tertiaires Telway du constructeur Télémécanique, réseaux installés sur les deux filières de traitement et dont la fonction est la communication interautomates.

Le réseau secondaire Modbus permet en particulier aux postes de supervision d'acheminer plus d'un millier d'informations dont une centaine en continu. Une alarme est ainsi disponible moins de deux secondes après son apparition dans la salle de commande, ce qui permet un diagnostic rapide et précis d'un éventuel problème qui peut survenir de jour comme de nuit. Le schéma complet de l'architecture du sytème informatique est présenté dans la figure ci-dessus.

\section{Les étapes de l'informatisation et les fonctions associées}

\section{* L'automatisation}

Les automates sont synchronisés sur deux réseaux tertiaires Telway. Chaque réseau a la charge d'une des deux filières de l'usine appelée Basse-Goulaine 1 (BG1) et BasseGoulaine 2 (BG2). Chaque filière de traitement est équipée 
de sept automates pour toutes les étapes du procédé. En outre, la filtration est équipée de "Filtronic" (automates brevetés SAUR pour la régulation du niveau des filtres) qui sont au nombre de 10 sur BG1 et 5 sur BG2 (1 Filtronic pour 1 filtre). Au total, l'installation possède 29 automates locaux qui assurent la partie opérationnelle du pilotage du traitement par le contrôle, la régulation et la gestion de chaque étape du procédé : l'exhaure, la reprise, l'asservissement aux réactifs, la filtration et la chloration. En ce qui concerne la filtration, il est à noter que l'automatisation du lavage des filtres se fait en mode multiplexage standard SAUR.

\section{* La supervision}

Les automates sont reliés aux deux postes de supervision en direct (chacun permettant l'envoi de réglages et supervisant une filière de traitement), grâce au réseau secondaire Modbus. La supervision assure d'une part le traitement en temps réel des informations locales acquises, et d'autre part, l'exploitation en temps différé des mêmes données en effectuant des traitements spécifiques, tels que les chronogrammes, les tableaux de bord ou les bilans journaliers. L'unité de supervision permet ainsi une vision en temps réel et différé du fonctionnement de l'installation, une connaissance et un diagnostic rapides des anomalies, une aide à la décision et au pilotage des procédés.

\section{* Les équipements distants}

Douze satellites périphériques installés sur les réservoirs avals primaires et secondaires permettent d'assurer une télésurveillance de l'information distante du site de l'usine en effectuant la collecte et l'enregistrement des données et les traitements opérationnels généraux. En outre, la télésurveillance assure une fonction d'alarme. Le satellite surveille en permanence les informations dont il a la charge pour, dans le cas d'alarme, prévenir soit le poste central, soit directement des agents d'astreinte. Enfin, le satellite peut être en état de veille en étant en permanence à l'écoute de ses lignes de communications pour répondre à des appels provenant du central, d'un agent ou d'un autre satellite.

\section{* La télégestion}

Le poste central de télégestion GEREMI, conçu et réalisé par la Direction technique de SAUR, est en ensemble progiciel de " Gestion des Réseaux par Moyens Informatiques " qui assure le contrôle et la gestion complète de sites répartis. Ce progiciel spécialisé permet ainsi de passer de l'ère de la télésurveillance à celle de la télégestion multiprotocole. C'est un outil de communication entre des équipements de marques et de natures différentes (satellites de télésurveillance et automates) et d'assistance continue à la décision. Il réalise la surveillance centralisée d'installations éloignées et la gestion personnalisée des informations recueillies. Il comporte de nombreux programmes et peut en particulier assurer une consultation sur Minitel, une gestion des astreintes, une synthèse vocale en clair et des traitements centraux tels que tableurs ou grapheurs. Il offre à l'exploitant les moyens d'acquérir l'information du réseau et de détecter les anomalies.

Enfin, il est à noter qu'un tel système informatique de télégestion GEREMI permet la décentralisation de la gestion des réseaux et les secours inter-postes centraux. Il est évolutif et modulaire et peut supporter d'autres applications d'aide à la conduite de l'usine telles que la gestion des stocks ou les systèmes experts.

\section{* Le poste central d'archives et d'aide à l'exploitant}

Ce poste constitue le système central d'information qui permet d'archiver et consolider l'information acquise et traitée, et d'effectuer des traitements d'aide à la gestion de l'exploitation tels que les bilans mensuels, les traitements statistiques, la gestion de l'énergie électrique, la maintenance assistée. Toutes les valeurs acquises ou calculées en interne par le poste central peuvent être archivées. Toute valeur archivée est horodatée et référencée. Les buts principaux de l'archivage sont de permettre :

- le tracé des courbes pour visualiser l'évolution de certains paramètres de l'installation ;

- l'obtention d'historique sur telle ou telle information ou station ;

- la présentation d'informations sous forme de tableauxbilans ou sous toute autre forme.

Toutes les informations archivées sont conservées pendant un minimum de trois mois. L'exploitant pourra en outre définir la durée de conservation des bilans et historiques. Enfin, les informations acquises par le poste central sont éditées sur imprimante. Cela permet à l'exploitant de ressortir et conserver une trace écrite des informations significatives du réseau et de son installation. Ce système ouvert et évolutif est donc un outil privilégié d'aide à la décision pour l'optimisation de la gestion d'exploitation.

\section{Conclusion}

Les cinq étapes de la modernisation de l'usine à l'aide de l'informatique industrielle ont pris trois années, ce qui a permis d'offrir l'avantage d'une formation progressive du personnel d'exploitation en deux grandes étapes : l'initiation à l'automatisation, l'entraînement à la supervision et à la télégestion.

Grâce à un tel système informatique au service de l'exploitant, celui-ci n'est plus un simple contrôleur-opérateur de terrain, il devient un véritable gestionnaire de son installation aidé par un puissant outil qui veille jour et nuit sur le fonctionnement de l'usine. 